\title{
HÖLDER CONTINUOUS SOBOLEV MAPPINGS AND THE LUSIN N PROPERTY
}

\author{
ALEKSANDRA ZAPADINSKAYA
}

\begin{abstract}
We give a new proof for the result of J. Malý and O. Martio, stating that Hölder continuous mappings in $W^{1, n}$ satisfy the Lusin $\mathrm{N}$ property. We further generalize this result to a metric setting.
\end{abstract}

\section{Introduction}

In this note, we study the Lusin $\mathrm{N}$ property for Sobolev mappings. We say that a mapping $f: \mathbb{R}^{n} \rightarrow \mathbb{R}^{m}$ satisfies the Lusin $\mathrm{N}$ property, if every set of zero Lebesgue $n$-measure has a zero $n$-dimensional Hausdorff measure image under $f$. The validity of the Lusin $\mathrm{N}$ property for a Sobolev mapping $f: \mathbb{R}^{n} \rightarrow \mathbb{R}^{n}$ enables the application of the change of variable formula for integration and of the area formula [7]. This fact makes the study of the Lusin N property important.

Let us consider a Sobolev mapping $f \in W^{1, p}\left(\Omega ; \mathbb{R}^{m}\right)$, defined in a domain $\Omega \subset \mathbb{R}^{n}$, where $2 \leq n \leq m$. It is well known that $p>n$ implies the Lusin $\mathrm{N}$ property [9]. On the other hand, this property may fail in the case $p=n$ (see examples in [12] and [8, Section 5]). However, additional assumptions on $f$, such as monotonicity or Hölder continuity, may guarantee the Lusin $\mathrm{N}$ property even when $p=n$ [11], [12], [10], [8].

We consider the latter case, that is we assume that our mapping $f \in W^{1, n}\left(\Omega ; \mathbb{R}^{m}\right)$ satisfies a Hölder continuity condition

$$
|f(x)-f(y)| \leq C_{0}|x-y|^{\gamma}
$$

for all $x, y \in \Omega$, where $C_{0}>0$ and $0<\gamma<1$. We give a new shorter proof for the result in $[8$, Theorem $\mathrm{C}]$, where it was established for $n=m$.

Received June 18, 2014; received in final form July 2, 2014.

2010 Mathematics Subject Classification. 46E35, 26B35, 28A75, 28A78. 
TheOREM 1. Let $\Omega$ be a domain in $\mathbb{R}^{n}$ and $f \in W^{1, n}\left(\Omega ; \mathbb{R}^{m}\right), m \geq n \geq 2$, a Hölder continuous mapping. Then $\mathcal{H}^{n}(f(E))=0$ for each $E \subset \Omega$, such that $|E|=0$.

We do not know, whether this Hölder continuity assumption is sharp. However, given any $\alpha \in] 0,(n-1) / n\left[\right.$, there exists a mapping $f \in W^{1, n}\left(\mathbb{R}^{n} ; \mathbb{R}^{n}\right)$, violating the Lusin $\mathrm{N}$ property and having modulus of continuity no worse than

$$
\psi(t)=C_{0} \exp \left(-c \log ^{\alpha}\left(\frac{1}{t}\right)\right)
$$

with some $C_{0}, c>0$ [5, Example 1.3]. Note that the modulus of continuity (1) we assume is (2) with $c=\gamma$ and $\alpha=1$.

The method we use has its origins in [6], where quasiconformal mappings were considered. First applications of those ideas to more general non-injective Sobolev mappings can be found in [4].

Our proof gives a direct generalization to a metric setting, providing a new result, stated as follows (see Section 3).

Theorem 2. Let $Q \geq 1$ and let $(X$, dist, $\mu)$ be an Ahlfors $Q$-regular metric measure space, which supports the Q-Poincaré inequality for continuous functions. Suppose that $f \in N^{1, Q}(X ; V)$, with some Banach space $V$, is a Hölder continuous mapping. Then $\mathcal{H}^{Q}(f(E))=0$ for each $E \subset X$, such that $\mu(E)=0$.

To demonstrate the elegance of the proof in the Euclidean case, we give separate proofs for the two theorems.

\section{Proof of Theorem 1}

We start by introducing our basic notation. Given a set $A \subset \mathbb{R}^{n}$, we denote its $n$-dimensional Lebesgue measure by $|A|$. If $|A|<\infty$ and $f$ is a Lebesgue integrable mapping, we denote the average $\frac{1}{|A|} \int_{A} f$ of $f$ over the set $A$ by $f_{A} f$ or $f_{A}$. Next, $A+a$ with $A \subset \mathbb{R}^{n}$ and $a>0$ stands for the set $\{x \in$ $\left.\mathbb{R}^{n}: \operatorname{dist}(x, A)<a\right\}$. By $\operatorname{diam}(A)$ and $\chi_{A}$, we denote the diameter and the characteristic function of the set $A$, respectively. Given a point $x \in \mathbb{R}^{m}$ and a non-negative number $r, B(x, r)$ denotes an open ball centred in $x$ and having radius $r$. If $B=B(x, r)$ is a ball and $a$ is a positive number, the notation $a B$ stands for the ball $B(x, a r)$. We write $\mathcal{H}_{\delta}^{s}(A)$ with $s>0$ and $0<\delta \leq \infty$ for the $s$-dimensional Hausdorff content of a set $A$, while $\mathcal{H}^{s}(A)$ denotes its $s$ dimensional Hausdorff measure. Finally, $C$ denotes a positive constant, which may depend on data ( $n, m$ and the modulus of continuity of $f$ ) and differ from occurrence to occurrence. 
We need also a weighted Hausdorff content of a set $A$ given by

$$
\lambda_{\infty}^{s}(A)=\inf \left\{\sum_{i=1}^{\infty} c_{i}\left(\operatorname{diam} U_{i}\right)^{s}: c_{i} \geq 0 \text { and } \chi_{A} \leq \sum_{i=1}^{\infty} c_{i} \chi_{U_{i}}\right\}
$$

for $s>0$. It is known that there exists a constant $\beta>0$, such that $\mathcal{H}_{\infty}^{s}(E) \leq$ $\beta \lambda_{\infty}^{s}(E)$ for all sets $E$ (see, for instance, [3], Theorem 8.6 and Theorem 9.7).

Proof of Theorem 1. We denote the modulus of continuity of $f$ by $\psi(t)=$ $C_{0} t^{\gamma}$. We may clearly assume that $E$ is bounded and $\bar{E} \subset \Omega$. Let us consider a dyadic decomposition of $\mathbb{R}^{n}$. We denote by $\mathcal{Q}_{i}=\left\{Q_{i, 1}, Q_{i, 2}, \ldots\right\}$ the collection of cubes of generation $i \in \mathbb{N}$ with edge length $2^{-i}$, such that $\mathbb{R}^{n}=\bigcup_{j=1}^{\infty} Q_{i, j}$. For each $i, j \in \mathbb{N}$, there exist $2^{n}$ cubes, denoted by $Q_{i, j}^{1}, \ldots, Q_{i, j}^{2^{n}} \in \mathcal{Q}_{i+1}$, such that $Q_{i, j}=\bigcup_{q=1}^{2^{n}} Q_{i, j}^{q}$. Similarly, when $i \geq 2$, the unique cube $Q \in \mathcal{Q}_{i-1}$, such that $Q_{i, j} \subset Q$ is denoted by $\hat{Q}_{i, j}$.

Once $Q_{i, j}$ is such that $\hat{Q}_{i, j} \subset \Omega$, we define $f_{i, j}=f_{Q_{i, j}} \in \mathbb{R}^{m}$ and

$$
r_{i, j}=\max \left\{\left|f_{i, j}-f_{\hat{Q}_{i, j}}\right|, \max _{q=1, \ldots, 2^{n}}\left|f_{i, j}-f_{Q_{i, j}^{q}}\right|\right\} .
$$

We obtain the following estimate for $q \in\left\{1, \ldots, 2^{n}\right\}$, using the Poincaré and Jensen inequalities

$$
\begin{aligned}
\left|f_{i, j}-f_{Q_{i, j}^{q}}\right| & \leq f_{Q_{i, j}^{q}}\left|f-f_{i, j}\right| \leq 2^{n} f_{Q_{i, j}}\left|f-f_{i, j}\right| \\
& \leq C \operatorname{diam} Q_{i, j} f_{Q_{i, j}}|D f| \\
& \leq C\left(\int_{Q_{i, j}}|D f|^{n}\right)^{1 / n} .
\end{aligned}
$$

Similar computations give $\left|f_{i, j}-f_{\hat{Q}_{i, j}}\right| \leq C\left(\int_{\hat{Q}_{i, j}}|D f|^{n}\right)^{1 / n}$. Thus, $r_{i, j}^{n} \leq$ $C \int_{\hat{Q}_{i, j}}|D f|^{n}$. For each $r_{i, j}>0$, we need a family of balls

$$
\mathcal{B}_{i, j}=\left\{B_{i, j}^{k}=B\left(f_{i, j}, r_{i, j} / 2^{k}\right): k=0,1, \ldots\right\} .
$$

Fix an arbitrary $\varepsilon>0$ and a $\delta \in] 0, \varepsilon]$, such that $E+\delta \subset \Omega$ and

$$
\int_{E+\delta}|D f|^{n}<\varepsilon
$$

Additionally, we choose a number $i_{0} \in \mathbb{N}$, which satisfies $\sqrt{n} 2^{-i_{0}+1}<\delta$. We restrict the families $\mathcal{Q}_{i}, i=i_{0}, \ldots$, so that $\hat{Q} \subset E+\delta$, whenever $Q \in \mathcal{Q}_{i}$.

Let $x \in E$. We choose a sequence of cubes $\left(Q_{i}(x)\right)_{i=i_{0}}^{\infty}$, such that $Q_{i}(x) \in \mathcal{Q}_{i}$ and $x \in Q_{i}(x)$. We have $Q_{i}(x)=Q_{i, j(i, x)}$ for a suitable index $j(i, x) \in \mathbb{N}$. This 
sequence defines a sequence of centres $f_{i}(x)=f_{i, j(i, x)}, i=i_{0}, \ldots$, on the image side, which converges to $f(x)$; indeed,

$$
\left|f(x)-f_{i}(x)\right| \leq f_{Q_{i}(x)}|f(x)-f(y)| d y \leq \psi\left(\operatorname{diam} Q_{i}(x)\right) \rightarrow 0,
$$

when $i$ goes to infinity. Finally, we put $r_{i}(x)=r_{i, j(i, x)}$. Note that (3) implies

$$
r_{i}(x) \geq \max \left\{\left|f_{i}(x)-f_{i-1}(x)\right|,\left|f_{i}(x)-f_{i+1}(x)\right|\right\}
$$

for each $i=i_{0}+1, \ldots$.

We neglect the set $E_{0}=\left\{x \in E: f_{i}(x)=f(x)\right.$ for each $\left.i=i_{0}, i_{0}+1, \ldots\right\}$, because its image under $f$ is countable. For a point $x \in E \backslash E_{0}$, we define a large number $l_{0}(x) \in \mathbb{N}$ so that there are some of $f_{i}(x)$ with $i \geq i_{0}$ outside the ball $B\left(f(x), 2^{-l_{0}(x)+1}\right)$. Denoting $E_{l}=\left\{x \in E \backslash E_{0}: l_{0}(x) \leq l\right\}$, we have $E \backslash E_{0}=\bigcup_{l \in \mathbb{N}} E_{l}$ and $f\left(E \backslash E_{0}\right)=\bigcup_{l} f\left(E_{l}\right)$.

Fix $l_{1} \in \mathbb{N}$ and consider the set $E_{l_{1}}$. Let $x \in E_{l_{1}}$ and $l=4 l_{1}$. We find the smallest number $J \in \mathbb{N}, J \geq i_{0}$, such that $f_{j}(x) \in B\left(f(x), 2^{-l}\right)$ for all $j>J$. We have by (5)

$$
2^{-l} \leq\left|f(x)-f_{J}(x)\right| \leq \psi\left(\operatorname{diam} Q_{J}(x)\right)=C_{0} \sqrt{n^{\gamma}} 2^{-J \gamma},
$$

which implies $l \geq J \gamma-\log _{2}\left(C_{0} \sqrt{n^{\gamma}}\right) \geq J \gamma / 2$, if $i_{0}$ is initially picked so that $i_{0} \geq \frac{2}{\gamma} \log _{2}\left(C_{0} \sqrt{n^{\gamma}}\right)$. On the other hand, if we denote by $N$ the number of integers $k$ in the set $\left\{l_{1}, \ldots, l\right\}$, such that the annulus $A_{k}(x)=B\left(f(x), 2^{-k+1}\right) \backslash$ $B\left(f(x), 2^{-k}\right)$ contains more than $8 / \gamma$ centres $f_{i}(x), i=i_{0}, \ldots, J$, we obtain $8 N / \gamma \leq J \leq 2 l / \gamma$, hence $N \leq l_{1}$. Thus, there exist at least $l-l_{1}+1-N \geq$ $2 l_{1}$ annuli $A_{k}(x), k=l_{1}, \ldots, l$, which contain at most $8 / \gamma$ centres $f_{i}(x)$, $i=i_{0}, \ldots, J$.

Let $A_{k}(x)$ be one such annulus. If it contains at least one centre $f_{i}(x)$ for some $i=i_{0}, \ldots, J$, then $(6)$ and the fact that $l_{1} \geq l_{0}(x)$ yield

$$
\sum_{f_{i}(x) \in A_{k}(x)} 2 r_{i}(x)>2^{-k} .
$$

Thus, there must be at least one $i \geq i_{0}$, such that $f_{i}(x) \in A_{k}(x)$ and $r_{i}(x) \geq$ $2^{-k-4} \gamma$. We have $f(x) \in \frac{32}{\gamma} B_{i, j(i, x)}^{0}=B\left(f_{i}(x), 32 r_{i}(x) / \gamma\right)$. Whenever there are no $f_{i}(x) \in A_{k}(x)$, we take the smallest $i=i_{0}, \ldots, J+1$ such that $f_{i}(x) \in$ $B\left(f(x), 2^{-k}\right)$. By $(6)$ and $l_{1} \geq l_{0}(x)$, we necessarily have $r_{i}(x)>2^{-k}$. We pick $B_{i, j(i, x)}^{p} \ni f(x)$ so that $2^{-k}<r_{i}(x) 2^{-p} \leq 2^{-k+1}$. Note that when $x$ is fixed and we choose balls for different $k$, each ball is taken no more than twice. That is

$$
2 \sum_{i=i_{0}}^{2 l / \gamma+1} \sum_{Q_{i, j} \in \mathcal{Q}_{i}} \sum_{k=0}^{\infty} \chi_{\frac{32}{\gamma} B_{i, j}^{k}}(y) \geq 2 l_{1}
$$


for each $y \in f\left(E_{l_{1}}\right)$. In other words, the collection of inflated balls $\frac{32}{\gamma} B_{i, j}^{k}$ covers the set $f\left(E_{l_{1}}\right)$ with $l_{1}$ layers. We conclude

$$
\begin{aligned}
\mathcal{H}_{\infty}^{n}\left(f\left(E_{l_{1}}\right)\right) & \leq \beta \lambda_{\infty}^{n}\left(f\left(E_{l_{1}}\right)\right) \leq \frac{\beta}{l_{1}} \sum_{i=i_{0}}^{2 l / \gamma+1} \sum_{Q_{i, j} \in \mathcal{Q}_{i}} \sum_{k=0}^{\infty} \frac{2^{6 n}}{\gamma^{n}} \frac{r_{i, j}^{n}}{2^{k n}} \\
& \leq \frac{C \beta}{l_{1}} \sum_{i=i_{0}}^{2 l / \gamma+1} \sum_{Q_{i, j} \in \mathcal{Q}_{i}} r_{i, j}^{n} \leq \frac{C \beta}{l_{1}} \sum_{i=i_{0}}^{2 l / \gamma+1} \sum_{Q_{i, j} \in \mathcal{Q}_{i}} \int_{\hat{Q}_{i, j}}|D f|^{n} \\
& \leq \frac{C \beta}{l_{1}} \sum_{i=i_{0}}^{2 l / \gamma+1} \int_{E+\delta}|D f|^{n} \leq \frac{C \beta\left(\frac{2 l}{\gamma}+1\right)}{l_{1}} \varepsilon \leq C \beta \varepsilon
\end{aligned}
$$

Since the sets $E_{l}$ are nested, we obtain $\mathcal{H}_{\infty}^{n}\left(f\left(E \backslash E_{0}\right)\right)<C \beta \varepsilon$. By the arbitrariness of $\varepsilon$, we have $\mathcal{H}_{\infty}^{n}\left(f\left(E \backslash E_{0}\right)\right)=0$, which yields $\mathcal{H}^{n}(f(E))=0$.

\section{Metric setting}

For this section, we preserve the notation $f_{A}=f_{A} f, \operatorname{diam}(A), \chi_{A}, B(x, r)$, $a B, \mathcal{H}_{\delta}^{s}(A), \mathcal{H}^{s}(A), \lambda_{\infty}^{s}(A)$, defined suitably. Recall that $\mathcal{H}_{\infty}^{s}(A) \leq \beta \lambda_{\infty}^{s}(A)$.

By Ahlfors regularity in the statement of Theorem 2, we mean that a metric space $\left(X\right.$, dist) is equipped with a Borel regular measure $\mu$, such that $c_{1} r^{Q} \leq$ $\mu(B) \leq c_{2} r^{Q}$, for all open balls $B \subset X$ of radius $\left.r \in\right] 0$, diam $X$ [ and some constants $Q, c_{1}, c_{2}>0$. Additionally, we assume that $(X$, dist, $\mu)$ supports $Q$-Poincaré inequality for continuous functions (see [2, Section 4]):

$$
f_{B}\left\|f-f_{B}\right\| d \mu \leq C_{P}(\operatorname{diam} B)\left(f_{\sigma B} \rho^{Q} d \mu\right)^{1 / Q}
$$

for all balls $B \subset X$, all continuous integrable functions $f$, defined in the ball $\sigma B$ and taking values in some Banach space $V$, all $Q$-weak $V$-upper gradients $\rho$ of $f$, and with constants $C_{P} \geq 0, \sigma \geq 1$, independent of $B, f$ and $\rho$. Let $V$ be a Banach space. The mapping $f$ in the statement of Theorem 2 is in the Sobolev class $N^{1, Q}(X ; V)$ (see [2, Section 3]) and is Hölder continuous with modulus $\psi(t)=C_{0} t^{\gamma}$. We fix some $Q$-weak $V$-upper gradient $\rho$ of $f$.

Proof of Theorem 2. The proof of Theorem 2 is a direct generalization of the proof in the previous section, so we just outline the main differences. Let us fix $\varepsilon>0$ and take an open set $\Omega \supset E$, such that

$$
\int_{\Omega} \rho^{Q}<\varepsilon
$$

Pick $i^{\prime} \in \mathbb{N}$ so that $10 \sigma 2^{-i^{\prime}}<\operatorname{diam} X$. This choice ensures that the radii of all balls, to which we are going to apply the doubling condition, are smaller than $\operatorname{diam} X$. We consider the decomposition $E=\bigcup_{i \geq i^{\prime}} E_{i}$, where $E_{i}=\{x \in$ $\left.E: B\left(x, 5 \sigma 2^{-i}\right) \subset \Omega\right\}$. Next, we fix an integer $i_{0} \geq i^{\prime}$ and consider the set $E_{i_{0}}$. 
Applying the covering theorem [1, Theorem 1.16], we obtain finite collections of balls $\mathcal{Q}_{i}, i=i_{0}, \ldots$, such that $E_{i_{0}} \subset \bigcup_{B \in \mathcal{Q}_{i}} B$, each $B \in \mathcal{Q}_{i}$ is centred in $E_{i_{0}}$ and has radius $2^{-i}$, and $\left\{\frac{1}{5} B: B \in \mathcal{Q}_{i}\right\}$ is a disjoint family for each $i=i_{0}, \ldots$

Let $x \in E_{i_{0}}$. There exists a sequence of balls $\left(B_{i}(x)\right)_{i=i_{0}}^{\infty}$, such that $x \in$ $B_{i} \in \mathcal{Q}_{i}$. We denote $f_{i}(x)=f_{B_{i}(x)}$. As in (5), we have $\left\|f_{i}(x)-f(x)\right\| \leq$ $\psi\left(2 \cdot 2^{-i}\right) \rightarrow 0$, when $i \rightarrow \infty$. Moreover, similarly to (4), we obtain

$$
\max \left\{\left\|f_{i}(x)-f_{i-1}(x)\right\|,\left\|f_{i}(x)-f_{i+1}(x)\right\|\right\} \leq A\left(\int_{5 \sigma B_{i}(x)} \rho^{Q}\right)^{1 / Q}
$$

for $i=i_{0}+1, \ldots$, where the constant $A>0$ depends on $c_{1}, c_{2}, Q, C_{P}$ and $\sigma$. We put $r_{B}=A\left(\int_{5 \sigma B} \rho^{Q}\right)^{1 / Q}$ for each $B \in \mathcal{Q}_{i}$ and each $i=i_{0}, \ldots$, and consider the collection $\mathcal{B}_{B}=\left\{B_{B}^{k}=B\left(f_{B}, r_{B} / 2^{k}\right): k=0,1, \ldots\right\}$.

As in the previous section, we decompose $E_{i_{0}}=\bigcup_{l} E_{i_{0}, l}$ according to the number $l_{0}(x)$ and fix some $E_{i_{0}, l_{1}}$ and $l=4 l_{1}$. Analogous argument implies

$$
2 \sum_{i=i_{0}}^{2 l / \gamma+1} \sum_{B \in \mathcal{Q}_{i}} \sum_{k=0}^{\infty} \chi_{\frac{32}{\gamma} B_{B}^{k}}(y) \geq 2 l_{1}
$$

for each $y \in f\left(E_{i_{0}, l_{1}}\right)$. Since the families $\left\{\frac{1}{5} B: B \in \mathcal{Q}_{i}\right\}$ are disjoint, the doubling condition for the measure $\mu$ gives the boundedness of the overlap

$$
\sum_{B \in \mathcal{Q}_{i}} \chi_{5 \sigma B}(y) \leq C
$$

for each $i=i_{0}, \ldots, y \in \Omega$ and some constant $C$, which depends on $c_{1}, c_{2}, Q$ and $\sigma$. We finally obtain similarly to $(7)$

$$
\mathcal{H}_{\infty}^{Q}\left(f\left(E_{i_{0}, l_{1}}\right)\right) \leq \frac{C}{l_{1}} \sum_{i=i_{0}}^{2 l / \gamma+1} \sum_{B \in \mathcal{Q}_{i}} \int_{5 \sigma B} \rho^{Q} \leq \frac{C}{l_{1}} \sum_{i=i_{0}}^{2 l / \gamma+1} \int_{\Omega} \rho^{Q} \leq C \varepsilon,
$$

where the constant $C>0$, differing from occurrence to occurrence, depends only on $\beta, \gamma, c_{1}, c_{2}, Q, C_{P}$ and $\sigma$. Thus, we conclude $\mathcal{H}_{\infty}^{Q}\left(f\left(E_{i_{0}}\right)\right) \leq C \varepsilon$ and $\mathcal{H}_{\infty}^{Q}(f(E)) \leq C \varepsilon$, since the involved sequences of sets are nested.

Acknowledgments. The author thanks Pekka Koskela for inspiring this note and the referees for the helpful comments.

\section{REFERENCES}

[1] J. Heinonen, Lectures on analysis on metric spaces, Universitext, Springer, New York, 2001. MR 1800917

[2] J. Heinonen, P. Koskela, N. Shanmugalingam and J. T. Tyson, Sobolev classes of Banach space-valued functions and quasiconformal mappings, J. Anal. Math. 85 (2001), 87-139. MR 1869604

[3] J. D. Howroyd, On the theory of Hausdorff measures in metric spaces, Ph.D. thesis, University College London, 1994; available at http://www. soupoftheday.gold.ac.uk/ jhowroyd/jdhthesis.pdf. 
[4] A. Kauranen and P. Koskela, Boundary blow up under Sobolev mappings, Anal. PDE 7 (2014), 1839-1850. MR 3318741

[5] P. Koskela, J. Malý and T. Zürcher, Lusin's condition $N$ and modulus of continuity, Adv. Calc. Var. 8 (2015), 155-171. MR 3331698

[6] P. Koskela and S. Rohde, Hausdorff dimension and mean porosity, Math. Ann. 309 (1997), no. 4, 593-609. MR 1483825

[7] J. Malý, The area formula for $W^{1, n}$-mappings, Comment. Math. Univ. Carolin. 35 (1994), no. 2, 291-298. MR 1286576

[8] J. Malý and O. Martio, Lusin's condition ( $N)$ and mappings of the class $W^{1, n}$, J. Reine Angew. Math. 458 (1995), 19-36. MR 1310951

[9] M. Marcus and V. J. Mizel, Transformations by functions in Sobolev spaces and lower semicontinuity for parametric variational problems, Bull. Amer. Math. Soc. (N.S.) 79 (1973), 790-795. MR 0322651

[10] O. Martio and W. P. Ziemer, Lusin's condition (N) and mappings with nonnegative Jacobians, Michigan Math. J. 39 (1992), no. 3, 495-508. MR 1182504

[11] J. G. Rešetnjak, Certain geometric properties of functions and mappings with generalized derivatives, Sibirsk. Mat. Z̆. 7 (1966), 886-919. MR 0203013

[12] Y. G. Reshetnyak, The $N$ condition for spatial mappings of the class $W_{n, \text { loc }}^{1}$, Sibirsk. Mat. Zh. 28 (1987), no. 5, 149-153. MR 0924990

Aleksandra Zapadinskaya, Department of Mathematics, University of Pisa, Largo Bruno Pontecorvo 5, 56127 Pisa, Italy

E-mail address: azapadinskaya@mail.dm.unipi.it 